\title{
Saúde Mental e Qualidade de Vida de estudantes universitários
}

\author{
Luiz Antonio Nogueira-Martins' ${ }^{1}$, Maria Cezira Fantini Nogueira-Martins ${ }^{2}$ \\ 'Universidade Federal de São Paulo. São Paulo, Brasil. ORCID: 0000-0001-7877-6480.nogmartins.luizantonio@gmail.com \\ ${ }^{2}$ Instituto de Saúde da Secretaria de Estado da Saúde de São Paulo. São Paulo, Brasil. ORCID: 0000-0002-5772-6262. mariacezira@gmail.com
}

Sabe-se, a partir de estudos sobre o desenvolvimento psicológico, que, a cada papel novo que o ser humano precisa desempenhar, são nele desencadeadas angústias primitivas, equivalentes àquelas que ocorrem no início da vida. Sabe-se, também, que o bebê passa por angústias advindas de impressões sensoriais e experiências emocionais, que precisam ser digeridas e devolvidas a ele como elementos toleráveis. No caso da criança, essa "digestão" é desempenhada pelo ambiente (habitualmente pela mãe). Essa capacidade de dar continência às vivências angustiantes, devolvendo ao bebê elementos "desintoxicados", constitui a base para o advento do pensamento. Portanto, o pensamento e o aprendizado estão calcados nas vivências emocionais experimentadas pela criança.

O aluno que ingressa na universidade, à semelhança da criança recém-nascida, enfrenta um mundo novo, desconhecido, ameaçador. Vai precisar de ajuda e amparo para superar essa etapa e seguir adiante. Vai precisar também de um ambiente de aprendizagem acolhedor, para desenvolver seu aprendizado.
Assim, consideramos que a vivência como aluno universitário constitui transição significativa na vida do indivíduo, pois ele está iniciando um novo papel. Essa transição inclui: o esquema de estudo (diferente do colégio e do cursinho); as provas e exames; a exigência de longas horas de estudo; os conflitos entre dever e lazer; a gestão do tempo; o sentimento de desamparo do estudante em relação ao poder dos professores (com a percepção, em algumas situações, do uso arbitrário deste poder); o estabelecimento de novos vínculos afetivos; a modificação dos métodos pedagógicos; a competição entre os estudantes; as expectativas quanto à carreira profissional; as dúvidas e preocupações sobre sua capacidade de absorver as informações dadas ao longo do curso; a preocupação com seus ganhos econômicos no futuro. Para alguns estudantes há, também, outras variáveis, tais como: - distanciamento da família; a ruptura de relações afetivas e sociais significativas; a mudança de cidade ou de estado; o estabelecimento de uma nova moradia. Para outros, ainda, há fatores relacionados a situações de vulnerabilidade pessoal, social e/ou econômica. 
Todos esses fatores podem levar ao sofrimento psíquico, comprometendo a saúde mental e a qualidade de vida dos alunos.

A relevância do estresse acadêmico no ensino superior pode ser atestada pela extensa produção científica recente disponível sobre o tema tanto na literatura internacional como na nacional. (Padovani et al., 2014; Harrod et al., 2014; Pedrelli, 2015; Fernandez et al., 2016; Graner et al., 2017; Newman et al., 2017; Wang et al., 2017; Kaloeti et al., 2018; Mortier et al., 2018; Ribeiro et al., 2018) Alguns temas abordados por esses estudos são: qualidade de vida; transtornos mentais comuns; estresse; burnout; ansiedade; depressão; ideação suicida; suicídio; uso de substâncias psicoativas; resiliência; distúrbios do sono.

Vale aqui ressaltar que as experiências durante 0 curso universitário são vivenciadas e elaboradas de forma distinta na dependência de diversos fatores. Um desses fatores, muito importante a ser considerado, diz respeito às características individuais do aluno (maior ou menor vulnerabilidade psicológica, facilidade/dificuldade de interação com colegas e professores, uso de estratégias de enfrentamento e mecanismos adaptativos).

Face ao exposto, como resultante da interação estudante-ambiente acadêmico, três situações podem ocorrer:

1. Adaptação. É um processo no qual acontece algum sofrimento psíquico transitório, sem repercussão importante na vida pessoal e acadêmica;

2. Crises adaptativas. São crises desencadeadas por conflitos emocionais relativos ao desenvolvimento pessoal e a eventos estressantes. São vinculadas tanto a conflitos próprios da idade e da vida pessoal como gestão do tempo, início/ consolidação/ruptura de relações afetivas como a conflitos interpessoais com professores e colegas e dificuldades socioeconômicas;

3. Crises psicopatológicas. São crises nas quais as dificuldades pessoais e acadêmicas ocorrem em estudantes mais vulneráveis, que padecem de transtornos mentais.
Com o objetivo de integrar as observações oriundas da experiência em serviços de atenção à saúde mental dos estudantes com observações embasadas na experiência docente, entendemos que o estresse vivenciado pelo estudante e seus problemas associados são decorrentes tanto do ambiente de aprendizagem como da vulnerabilidade psicológica do estudante e podem ser agrupados em três categorias:

1. Estresse relacionado ao processo de profissionalização - ligado às vicissitudes da construção de uma carreira/identidade profissional em uma realidade de mercado de trabalho altamente competitivo;

2. Estresse situacional - associado às pressões e exigências específicas do treinamento na graduação (qualidade do ensino, provas, atendimento de pacientes), dificuldades nos relacionamentos com colegas e professores;

3. Estresse pessoal - vinculado a características individuais e traços de personalidade como gênero, história pessoal e familiar de doenças físicas e transtornos mentais, vulnerabilidades psicológicas (maior ou menor dificuldade em lidar com situações de conflitos interpessoais), situação econômica e social (necessidades econômicas pessoais), problemas familiares e eventos estressantes da vida (doença e morte de pessoas próximas, crises familiares).

Considerando que as condições psicológicas são determinantes para $\circ$ processo de aprendizagem, as universidades não devem medir esforços no sentido da criação de uma atmosfera aberta e facilitadora, para atenuar o estresse ligado ao processo de profissionalização dos estudantes. Os professores, preceptores, enfim, toda a equipe de ensino pode criar esse ambiente de aprendizagem continente $e$ facilitador. 
Para a melhoria das condições de saúde mental e de qualidade de vida do estudante universitário, é importante levar em conta os dois tipos de crise e os três tipos de estresse citados. Assim, medidas preventivas devem abranger os níveis de prevenção primária (promoção de saúde e proteção específica), prevenção secundária (diagnóstico e tratamento precoces e limitação da incapacidade) e prevenção terciária (reabilitação e readequação ocupacional). Dentre elas, destacam-se:

- Serviço de assistência psicológica e psiquiátrica;

- Rastreamento e identificação precoce de transtornos mentais entre os alunos;

- Serviço de apoio pedagógico;

- Serviço de apoio social;

- Incentivo a pesquisas de iniciação científica sobre estresse acadêmico;

- Implantação de reformas curriculares com criação de tempo livre para o aluno;

- Utilização de recursos pedagógicos como Grupos de Reflexão;

- Desenvolvimento pedagógico de professores;

- Atividades de aproximação à vida profissional (palestras de profissionais; visitas a instituições);

- Oferta e incentivo a atividades culturais, esportivas e de lazer;

- Desenvolvimento de Programas de Tutoria (mentoring);

- Utilização de métodos ativos de ensino;

- Atenção ao ambiente de aprendizagem;

- Programas de recepção aos calouros;

- Programas de apadrinhamento de calouros por veteranos.

A identificação precoce das tendências de comportamentos do estudante frente às fontes de tensão do curso universitário assume um caráter importante diante dos possíveis riscos aos quais estão sujeitos os estudantes e que, na maioria das vezes, se estendem ao longo de sua vida profissional.

A atenção à saúde do estudante, e em especial à saúde mental, não deve ser apenas uma preocupação dos profissionais da área de saúde mental; essa questão deve envolver gestores, docentes, pedagogos, assistentes sociais e deve ser difundida e valorizada pelas instituições de ensino superior, abarcando o ambiente de aprendizagem como um todo.

\section{Referências}

Padovani, R. C., Neufeld, C. B., Maltoni, J., Barbosa, L. N. F., Souza, W. F., Cavalcanti, H. A. F., ... Lameu, J. N. (2014). Vulnerabilidade e bem-estar psicológicos do estudante universitário. Revista Brasileira de Terapias Cognitivas, 10(1), 2-10. Recuperado de http://pepsic. bvsalud.org/pdf/rbtcc/v1 On 1/v1 On 1 a02.pdf. doi: 10.5935/1808-5687.20140002

Harrod, C. S., Goss, C. W., Stallones, L., \& DiGuiseppi, C. (2014). Interventions for primary prevention of suicide in university and other post-secondary educational settings. Cochrane Database of Systematic Reviews, (10):CD009439. Recuperado de https:// www.ncbi.nlm.nih.gov/pubmed/25353703. doi: 10.1002/14651858.CD009439.pub2

Pedrelli, P., Nyer, M., Yeung, A., Zulauf, C., \& Wilens, T. (2015). College Students: Mental Health Problems and Treatment Considerations. Academic Psychiatry, 39(5), 503-511. Recuperado de https://www.ncbi.nlm.nih. gov/pmc/articles/PMC4527955/. doi: 10.1007/ s40596-014-0205-9

Fernandez, A., Howse, E., Rubio-Valera, M., Thorncraft, K., Noone, J., Luu, X., ... Salvador-Carulla, L. (2016). Setting-based interventions to promote mental health at the university: a systematic review. International Journal of Public Health, 61 (7), 797-807. doi: 10.1007/s00038-016-0846-4

Graner, K. M., \& Ramos-Cerqueira, A. T. A. (2017). Revisão integrativa: Sofrimento psíquico em estudantes universitários e fatores associados. Ciência \& Saúde Coletiva. Recuperado de http://www. cienciaesaudecoletiva.com.br/artigos/revisaointegrativa-sofrimento-psiquico-em-estudantesuniversitarios-e-fatores-associados $/ 16374$ ? id $=16374$

Newman, I., Ding, L., \& Feng, Y. (2017). Estimate of undergraduate university student alcohol use in China: a systematic review and meta-analysis. Archives of Public Health, 75:52. Recuperado de https://www. ncbi.nlm.nih.gov/pmc/articles/PMC5724242/. doi: 10.1186/s13690-017-0220-x

Wang, Y. H., Shi, Z. T., \& Luo, Q.Y. (2017). Association of depressive symptoms and suicidal ideation among university students in China: A systematic review and meta-analysis. Medicine (Baltimore), 96(13), e6476. Recuperado de https://www.ncbi. nlm.nih.gov/pubmed/28353586. doi: 10.1097/ MD.0000000000006476 
Kaloeti, D. V. S., Rahmandani, A., Sakti, H., Salma, S., Suparno, S., \& Hanafi S. (2018). Effect of childhood adversity experiences, psychological distress, and resilience on depressive symptoms among Indonesian university students. International Journal of Adolescence and Youth. Recuperado de https://www.tandfonline. $\mathrm{com} / \mathrm{doi} /$ full/10.1080/02673843.2018.1485584. doi: $10.1080 / 02673843.2018 .1485584$

Mortier, P., Cuijpers, P., Kiekens, G., Auerbach, R. P., Demyttenaere, K., Green, J. G., ... Bruffaerts, R. (2018). The prevalence of suicidal thoughts and behaviours among college students: a meta-analysis. Psychological Medicine, 48(4), 554-565. Recuperado de https://www.ncbi.nlm.nih.gov/pubmed/28805169. doi: $10.1017 /$ S0033291717002215

Ribeiro, I. J. S., Pereira, R., Freire, I. V., Oliveira, B. G., Casotti, C. A., Boery, E. N. (2018). Stress and Quality of Life Among University Students: A Systematic Literature Review. Health Professions Education. Recuperado de https://www.researchgate.net/ publication/316264902_Stress_and_Quality_of_Life_ Among_University_Students_A_Systematic_Literature_ Review. doi: 10.1016/i.hpe.2017.03.002 